\title{
THE BONN SPECTRAL ATLAS: PART II
}

\author{
W. C. SEITTER \\ Five College Astronomy Dept., U.S.A., and Bonn University Observatory, Germany
}

\begin{abstract}
Summary. When work commenced on the Bonn Atlas for Objective Prism Spectra some years ago it was our intention to supply a tool for astronomers working with such widely different dispersions and resolutions as are used in the field of objective prism spectroscopy. This was to be accomplished through an atlas which contained and compared spectra of considerable difference in dispersion.

The Bonn Schmidt telescope which is used to obtain the observational material is equipped with three prisms giving linear reciprocal dispersions of 240,645 and $1280 \AA \mathrm{mm}^{-1}$ at $\mathrm{H} \gamma$, thus covering a good part of the range generally used.

The first part of the atlas, containing spectra of the largest dispersion only, was published as soon as it was finished, while work on the second part with the two lower dispersions was still in progress. Thus, one of the main intended features of the complete publication, a comparison of criteria displayed at different dispersions, with a special interest in the appearance and disappearance of certain criteria as one goes from higher to lower dispersions, was not yet possible.
\end{abstract}

Now, work on the second part of the atlas has progressed far enough for sample pages of the lower dispersion plates to be distributed for inspection and discussion. Plate I gives examples of spectra that will be used on the S-plates (sequences of different spectral types for a given luminosity class) showing both spectra of 645 $\AA \mathrm{mm}^{-1}$ (right hand side) and $1280 \AA \mathrm{mm}^{-1}$ (left hand side).

While the first part of the atlas tried to point out as much detail in the spectra. as possible to make the atlas applicable for work with even higher dispersions, the second part concentrates only on those features which are important in the mediumto low-resolution range.

Originally, it was intended to use greater enlargements on the L-plates (sequences of different luminosity classes for a given spectral type). Yet, general agreement upon inspection of the sample plates, which are not shown here, was that the smaller enlargements are better and thus they will be used in the final version of the atlas.

A detailed explanation of the different spectral features useful in classification from medium- to low-dispersion plates will be possible only after completion of all observational work. So far it is indicated, as was to be expected from the work of other authors, that some of the most useful luminosity criteria overlap seriously with population criteria, e.g. the $\mathrm{CN}$ bands.

The discussion at the symposium revealed an interest in the sampling of peculiar spectra. Following this suggestion work has began on preparing for a third part of the atlas which is planned to contain spectra of about 70 peculiar stars taken with all three above-mentioned dispersions. 


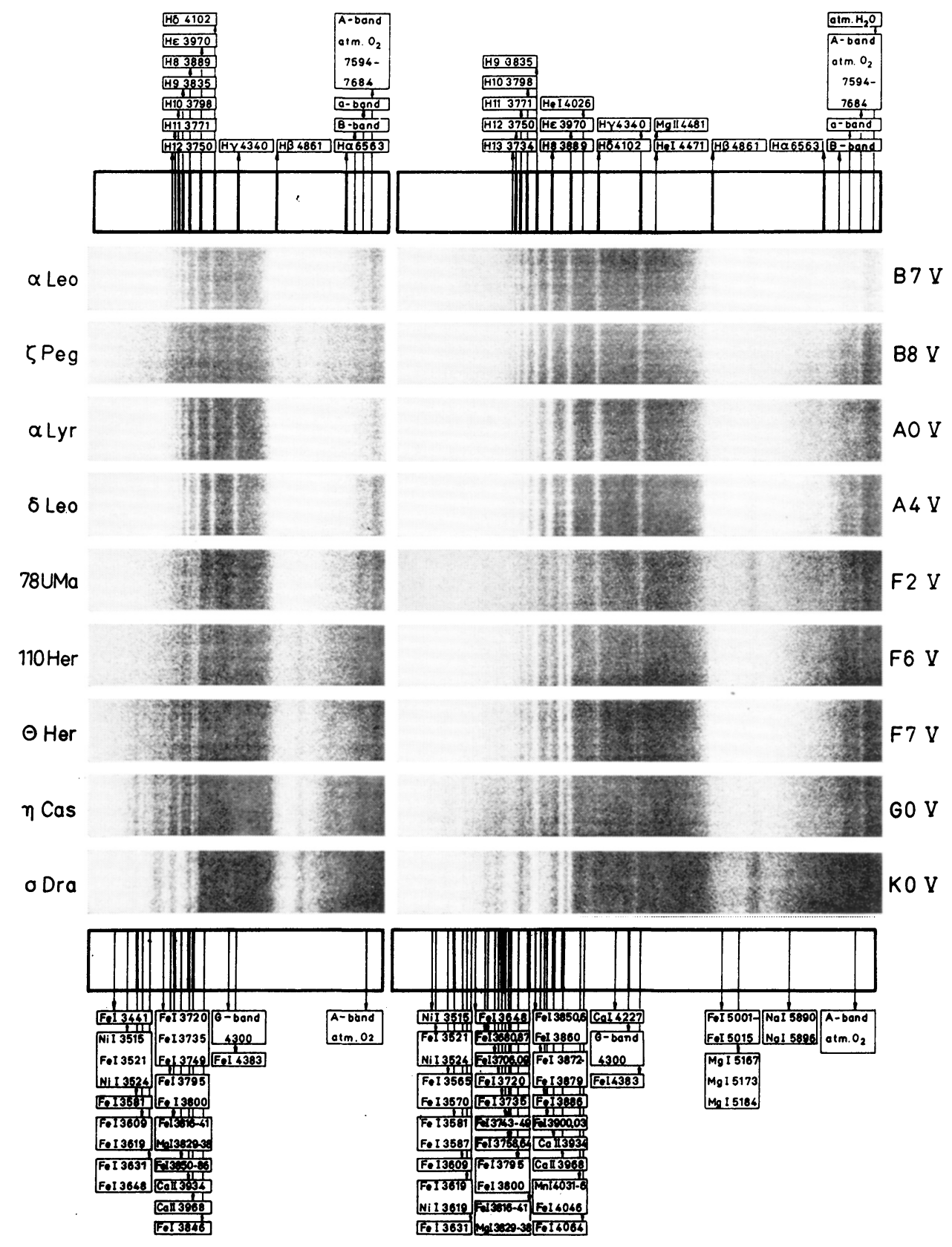

Plate I. 